\title{
Interaction Between Perceived and Imagined Rotation
}

\author{
Michael C. Corballis and Rachael McLaren \\ University of Auckland, Auckland, New Zealand
}

\begin{abstract}
In Experiment 1, subjects performed a mental-rotation task in which they were timed as they decided whether rotated letters were normal or backwards. Between presentations of the letters, they watched a rotating textured disk that induced an aftereffect of rotary movement on the letters. The function relating reaction times to orientation was influenced asymmetrically by the aftereffect, suggesting that perceived movement interacts with imagined movement. Experiment 2 showed that the aftereffect produced a negligible influence on perceived orientation, suggesting that the influence of the aftereffect on mental rotation was not caused by changes in the perceived orientations of the letters. Detailed analysis of the mental-rotation functions suggested that the aftereffect may sometimes have induced subjects to rotate letters through the larger rather than the smaller angle back to the upright where the aftereffect was in the appropriate direction.
\end{abstract}

Mental rotation is the act of imagining some object or pattern turning from one angular orientation to another. In some respects, imagining a pattern rotating is like seeing it actually rotating. Both take place in real time in the sense that it takes longer to rotate a pattern the larger the angle it is rotated through, and both take place in three-dimensional space (Shepard \& Metzler, 1971). Moreover, Cooper and Shepard (1973) have demonstrated striking correspondences between imagining a letter or a digit in a certain orientation and actually perceiving it in that orientation. In their paradigm, subjects made timed decisions as to whether a rotated alphanumeric character was normal or backward (i.e., mirror-reversed). Decision times increased monotonically with the angular departure of the characters from the upright, suggesting that the subjects mentally rotated the characters back to the upright before making their decisions. If the subjects knew the identity and orientation of each character sufficiently in advance of its presentation, however, then

The research was supported by a special grant from the New Zealand Optometrical Association and by grant No. 140 Psychology 22 from the New Zealand University Grants Committee.

We thank Sharon Cullen and Adrian Gane for help with the experiments.

Requests for reprints should be sent to Michael $C$. Corballis, Department of Psychology, University of Auckland, Private Bag, Auckland, New Zealand. decision time as measured from the onset of the character was more or less independent of its orientation. That is, the subjects could evidently prepare a rotated image of the pattern in advance of its presentation and then compare the actual pattern directly with this rotated image. Cooper and Shepard also had subjects mentally rotate a character in accordance with verbal prompts specifying successive angular positions, and again found that the subjects could compare a presented version of that character directly with the rotated image. At some level, then, image and percept appear to occupy some common internal "space."

These experiments demonstrate a correspondence between the perceived and imagined positions of an alphanumeric character but leave unproven whether there is any common ground between perceived and imagined movement. Introspectively, mental rotation does appear to possess the smooth, continuous quality of true rotation, but as Cooper and Shepard (1973) recognized their experimental data are just as consistent with the notion that mental rotation is achieved by a series of discrete computations of successive angular positions. Introspection is not an infallible guide, of course, and might itself be based on inference rather than on direct access to cognitive mechanisms. In the present study, therefore, we sought more direct evidence for an interaction between perceived and imagined rotation. 
Our procedure was to display disoriented letters and have subjects judge them as normal or as backward after we induced an aftereffect of rotation on each letter. We induced this effect by having the subjects watch a rotating disk between presentations of the letters, so that they perceived the letters to be rotating in the direction opposite to that of the disk. This motion aftereffect seemed uniquely tailored to our purpose of studying the effects of perceived motion separately from the effects of perceived position, since it is commonly asserted that a pattern that is subjected to a motion aftereffect is seen paradoxically to move without changing its position (e.g., MacKay, 1973; Rock, 1975; Wohlgemuth, 1911). Consequently, any effect of a rotation aftereffect on mental rotation could presumably be attributed to the influence of perceived motion and not to changes in perceived position. Our general expectation was that mental rotation would be facilitated when the aftereffect was in the same direction as the mental rotation and inhibited when the two were in opposite directions.

\section{Experiment 1}

\section{Method}

\section{Subjects}

The subjects were 48 volunteers $(24$ men and 24 women) aged 17-42 years, all with normal or corrected vision.

\section{Stimuli and Apparatus}

The stimuli were presented in a Gerbrands four-field tachistoscope. A white disk, $16 \mathrm{~cm}$ in diameter, with black letters and numerals placed in random positions and orientations on it to give a textured appearance, was placed in one field and could be rotated in either direction. The letters to be judged normal or backward were the uppercase letters $F, G$, and $R$, which were placed in the center of white cards in either normal or backward versions at six different angular orientations, ranging in $60^{\circ}$ steps from $0^{\circ}$ to $300^{\circ}$ clockwise from the upright. Thus there were 36 possible stimuli, each duplicated to give a total of 72 cards. When presented in the tachistoscope, each letter subtended an angle of 21 ' lengthwise at the subject's eyes. The disk and the letters were displayed within a circular surround subtending a diameter of $8.82^{\circ}$.

'Presentation of the letters activated a timer, which stopped when the subjects' verbal responses triggered a voice key. This enabled decision latencies to be recorded to the nearest $.01 \mathrm{sec}$.

\section{Procedure}

On each trial, the rotating disk appeared for $9 \mathrm{sec}$, followed by a 100-msec presentation of a dark field, which served as a warning signal. The rotating disk then returned for $500 \mathrm{msec}$ followed by presentation of a letter. For half of the subjects ( 12 men, 12 women) the letter appeared for $2 \mathrm{sec}$ while for the remainder it appeared for $90 \mathrm{msec}$ followed by a dark field for $2 \mathrm{sec}$. In both cases the disk returned immediately to initiate the beginning of the next trial, so that the subjects were subjected to the rotating disk more or less continuously between presentations of the letters. For each stimulus duration, half of the subjects always saw the disk rotating at 25 revolutions per minute (rpm) while the other half saw it rotating at $60 \mathrm{rpm}$. Each subject was told to watch the center of the rotating disk to say "right" if the letter was normal when it appeared and "wrong" if it was backward. They were told to respond as quickly as they could without sacrificing accuracy.

The subjects watched the rotating disk for a minute or two before the letters were presented and were given several practice trials until they felt they thoroughly understood what was required of them. Most subjects then embarked on a series of 72 experimental trials, with the 72 cards presented in random order. If a subject made more than five errors in the first 36 trials, however, the series was discontinued and the subject replaced. Otherwise, cards on which a subject made errors were repeated at the end of the sequence, so that all subjects retained in the experiment provided 72 correct responses.

The direction of rotation of the disk was then reversed, and after a few minutes of exposure to the rotating disk, the subject was given a further 72 experimental trials, with cards on which errors occurred again repeated at the end of the sequence so that all subjects provided 72 correct responses. Clockwise and counterclockwise rotations of the disk were presented in counterbalanced order within each of the subgroups formed by the crossing of sex, stimulus durations, and rate of rotation of the disk.

\section{Results and Discussion}

Errors were fewer than $5 \%$ and were disregarded in the analysis. As explained above, all subjects were tested until they provided 72 correct responses under each direction of rotation of the disk, and latencies for these responses were subjected to analyses of variance (ANOVAS).

\section{Overall Analysis of Latencies}

First, an overall ANOva was carried out. Between-subjects variables were speed of rotation of the disk, order of presenting the different directions of rotation, duration of presentation of the letter, and sex of the subjects. Within-subjects variables were direction of rotation, "right" vs. "wrong" judg- 
ments, and angular orientation of the letters. Several main effects were significant: Mean reaction time (RT) was shorter for rotation of $25 \mathrm{rpm}(M=930 \mathrm{msec})$ than for $60 \mathrm{rpm}$ $(M=1,078 \mathrm{msec}), F(1,32)=5.47, p<.05$; shorter for counterclockwise rotation (i.e., clockwise aftereffect; $M=989 \mathrm{msec}$ ) than for clockwise rotation $(M=1,020 \mathrm{msec})$, $F(1,32)=14.32, p<.01$; and shorter for right judgments $(M=916 \mathrm{msec})$ than for wrong judgments $(M=1,093 \mathrm{msec}), F(1$, $32)=115.73, p<.01$. There was also a significant main effect due to orientation, $F(5$, $160)=84.49, p<.01$.

Of more immediate interest to the purpose of the experiment was the significant interaction between direction of rotation of the disk and angular orientation of the letters, $F(5,160)=29.41, p<.01$, suggesting that mental rotation was influenced by the motion aftereffect. These two factors also featured in significant three-way interactions, however, with speed of rotation, $F(5,160)=$ $3.40, p<.01$, and with duration of presen- tation of the letters, $F(5,160)=3.37, p<$ .01 , and in a significant four-way interaction with both speed and duration, $F(5,160)=$ $5.63, p<.01$ (although only the last of these significant at the .05 level if one adopts the conservative degrees of freedom $[1,32]$ recommended by Winer, 1971, in testing repeated-measurement effects). Consequently, separate ANOVAS were carried out for each of the four groups of subjects who worked under the different combinations of rotation speed and letter duration. In each case the interaction between direction of rotation and angular orientation was significant $(p<.05)$, even according to conservative degrees of freedom. The interaction is plotted for each group in Figure 1.

Figure 1 shows the mental-rotation functions for each direction of the aftereffect, which is opposite the direction of rotation of the disk. It is clear that each function is distorted by the aftereffect, such that latencies are generally longer when the aftereffect is opposite to the presumed direction of men-
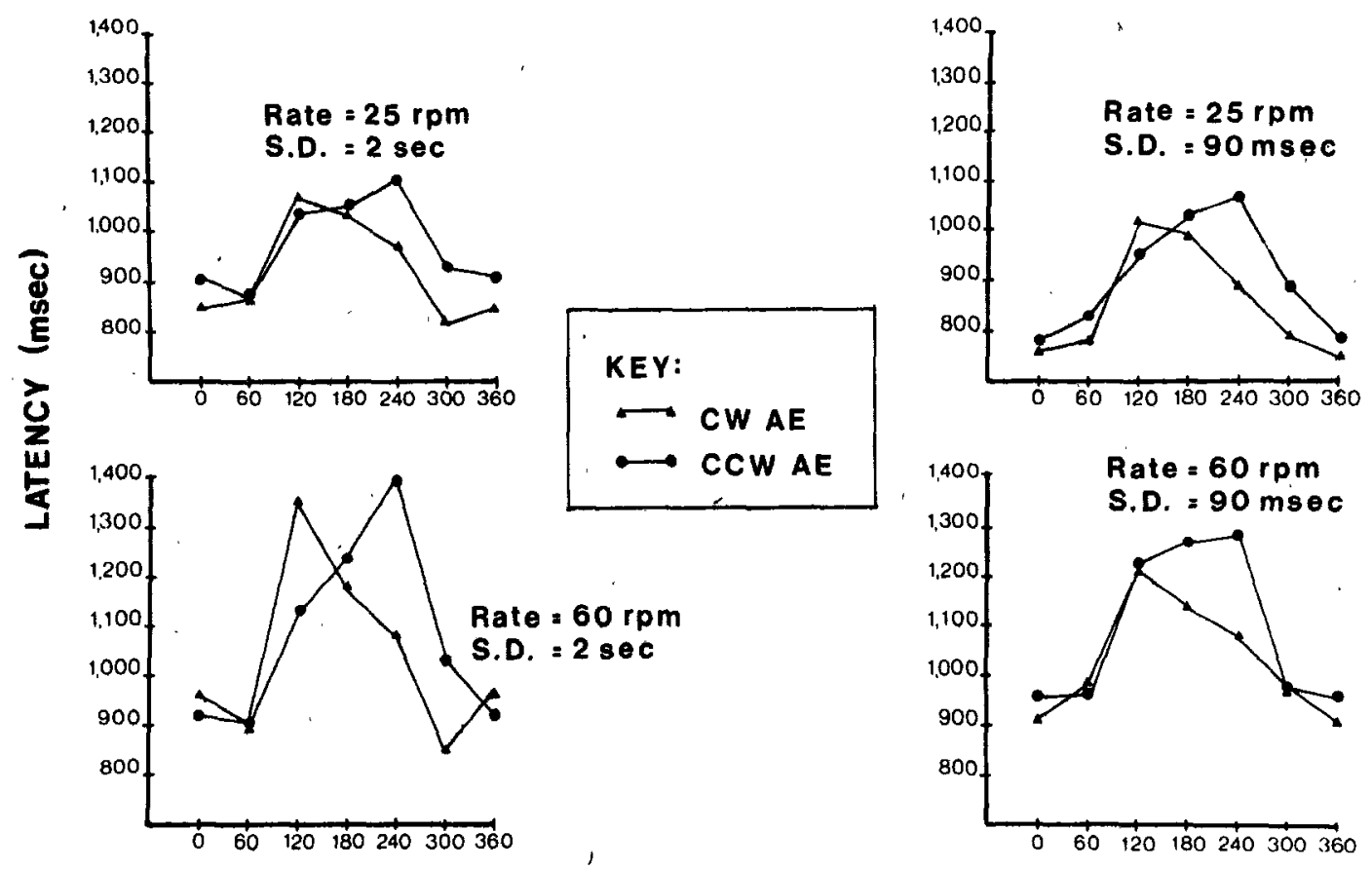

\section{LETTER ORIENTATION (degrees clockwise from vertical)}

Figure 1, Mean latencies for each orientation and each direction of aftereffect, shown separately for each rotation of disk and each stimulus duration (SD). (CW AE = clockwise aftereffect. $\mathrm{CCW} \mathrm{AE}=$ counterclockwise aftereffect.) 
tal rotation and, to a lesser extent, shorter when it is in the same direction as that of mental rotation. This demonstrates an interaction between perceived and imagined rotation.

We have considered three hypotheses as to how the aftereffect may have influenced mental rotation.

Hypothesis 1. One possibility is that the aftereffect may have caused the letters to be perceived as though rotated away from their actual orientations, in the direction of the aftereffect. That is, the functions might have been shifted laterally. This hypothesis runs counter to the doctrine that the motion aftereffect is characterized paradoxically by perceived movement but not by changes in perceived position (Wohlgemuth, 1911). Nevertheless, there is an aspect of the data that suggests that part of the effect may have been due to changes in perceived orientation. In all eight functions plotted in Figure 1, the maxima were shifted away from the $180^{\circ}$ point in the direction of the aftereffect, consistent with the notion that a letter that was actually at $180^{\circ}$ was seen as though closer to $120^{\circ}$ or $240^{\circ}$ (depending on the direction of the aftereffect). Conversely, a letter actually at $120^{\circ}$ or $240^{\circ}$ was seen as closer to $180^{\circ}$. Notice also that in four of the eight functions, the minima were also displaced in the direction of the aftereffect. Although these displacements were slight compared with those of the maxima, the only plausible explanation seems to be that the letters were seen as though rotated. Thus a letter that was actually upright was seen as though tilted, and it had to be mentally rotated to be perceived upright in performance of the task. Letters that were actually tilted $60^{\circ}$ were seen as closer to the upright than those that were actually upright, and consequently they were responded to more quickly.

It is also noteworthy, however, that the displacement of the minima occurred only in those conditions in which the letters were presented for $2 \mathrm{sec}$ (i.e., on the left-hand side of Figure 1). When the duration was cut back to $90 \mathrm{msec}$, the maxima were shifted but the minima were not. This short duration may have prevented the letters from being perceived in an altered orientation. The shift in the maxima in this condition, and perhaps the main part of the effect even under the longer stimulus duration, might then be attributed to a different kind of influence, as suggested by the following hypotheses.

Hypothesis 2. It is possible that the rate of perceived rotation due to the aftereffect might have combined additively with the rate of mental rotation, so that mental rotation was faster in the direction of the aftereffect and slower in the opposite direction. According to this hypothesis, the effect of the aftereffect would have been to shift the data points vertically (rather than laterally, as predicted by Hypothesis 1). According to this hypothesis, the shortest latencies should still have occurred when the letters were in fact upright. As Figure 1 shows, this was true for the 90-msec duration but not for the 2-sec duration, although the effects are rather slight. Indeed, this feature does not provide a very sensitive measure of which hypothesis is the more likely to be correct, since even in the absence of any aftereffect there is typically a flattening of the mentalrotation function for orientations close to the normal upright (Cooper \& Shepard, 1973). Nevertheless the functions for the $90-\mathrm{msec}$ duration do seem more consistent with $\mathrm{Hy}-$ pothesis 2 than with Hypothesis 1.

Hypothesis 3. Some subjects suggested that when the letter was $120^{\circ}$ from the upright they tended to mentally rotate it through the larger $240^{\circ}$ angle back to the upright when the aftereffect was in that direction. According to this hypothesis, the only influence of the aftereffect would be to increase the latency for either the $120^{\circ}$ point or the $240^{\circ}$ point, depending on the direction of the aftereffect. As Figure 1 shows, this characterization provides a reasonable description of the data.

\section{Comparing the Three Hypotheses}

It is of course possible that all three mechanisms outlined above contributed to the influence of the aftereffect. Nevertheless, we attempted to distinguish between them by fitting functions representing each hypothesis to the two orientation functions for each individual and then comparing goodness of fit across the three. The functions were defined as follows. 
For Hypothesis 1, suppose that by the time the subject initiated mental rotation, the letter was perceptually displaced $\theta^{\circ}$ from its actual orientation. If, say, $\theta$ represents a clockwise displacement and $0 \leq \theta \leq 60$, then for true orientations of $0^{\circ}, 60^{\circ}, 120^{\circ}, 240^{\circ}$, and $300^{\circ}$ we assumed the latencies to be proportional to $\theta,(60+\theta),(120+\theta),(180-$ $\theta),(120-\theta)$, and $(60-\theta)$, respectively. Similar expressions can be derived for possible values of $\theta$ within any of the other five sextants. We used the method of least squares to determine the best-fitting value of $\theta$ over all sextants, and to estimate the rate of mental rotation.

For Hypothesis 2, suppose that the rate of mental rotation (or imagined rotation) was some value $I$ and the perceived rate of the aftereffect was $P$, where $P$ is assumed clockwise and $P$ and $I$ are in degrees per sec. The latencies should be proportioned to 0 , $60 /(I-P), 120 /(I-P), 180 /(I+P), 240 /$ $(I+P)$, and $300 /(I+P)$. Again, we fitted this hypothetical function by the method of least squares and estimated $I$ and $P$.

For Hypothesis 3, let us illustrate the case in which the aftereffect is clockwise. We supposed that for true orientations of $0^{\circ}, 60^{\circ}$, $180^{\circ}, 240^{\circ}$, and $300^{\circ}$, latencies would be proportional to $0,60,180,120$, and 60 , respectively. For the $120^{\circ}$ orientation, we assumed that the subject mentally rotated the letter $240^{\circ}$ with some probability $p$, and $120^{\circ}$ with probability $(1-p)$, so that latency was proportional to $(240 p+120[1-p])$, or $120(1+p)$. Again, we used the method of least squares to estimate $p$ and $I$.

The functions are at least roughly comparable in that each involves the same number of parameters, although there are other constraints that render precise comparison of goodness of fit questionable. For Hypothesis 1 , we allowed $\theta$ to be either positive or negative, so that in some individual cases the best estimate of perceived position was opposite the direction of the affereffect. Similarly, for Hypothesis 2, we allowed negative estimates of $P$, implying that the aftereffect subtracted from rather than added to the mental rotation. For Hypothesis 3, the estimated value of $p$ was in some cases greater than unity. We allowed this, however, since the expression $(1+p)$ can be considered generally as an elevation factor rather than as an expression involving strict probability. The elevation in latency might be due in part to indecision as to which way to rotate the letter mentally, as well as to the added mental-rotation time to rotate it through the larger $240^{\circ}$ angle.

The proportion of variance attributable to each hypothesis for each subject under each duration of the aftereffect was transformed by the arc sine transformation (Winer, 1971) and subjected to ANOVA. The independent variables were rate of rotation, stimulus duration, sex, order, direction of rotation, and hypothesis. There was a significant main effect due to hypotheses, $F(2,64)=$ $5.58, p<.01$, with the highest proportion of variance attributable to Hypothesis $3(M=$ $.765)$, followed by Hypothesis $1(M=.744)$ and then Hypothesis $2(M=.727)$. Newman-Keuls post hoc tests revealed that $\mathrm{Hy}$ pothesis 3 accounted for significantly more variance $(p<.05)$ than the other two, which did not differ significantly from each other. There was, however, a significant triple interaction between rate of rotation, stimulus duration, and hypothesis, $F(2,64)=4.95$, $p<.05$. Inspection of this interaction revealed that the advantage of Hypothesis 3 was confined to the 2-sec stimulus duration under the slower rotation rate and to the 90msec stimulus duration under the faster rate. There seems no obvious interpretation for this interaction, but it does at least caution against concluding that Hypothesis 3 provides the best fit under all conditions.

Table 1 shows the mean percentages of variance accounted for and the mean parameter estimates under the three hypotheses, shown for each condition of rotation rate and stimulus duration. Although Hypothesis 3 provided the best overall fit, the parameter estimates under this hypothesis are not entirely satisfactory; two of the four mean probability estimates are greater than zero, and one of the four mean estimates of mental rotation $\left(1,800^{\circ} / \mathrm{sec}\right)$ is unreasonably high. Hypothesis 1 yielded a more "reasonable" set of parameter estimates.

We also subjected the parameter estimates to ANOVAs; the values of $\theta$ under $\mathrm{Hy}$ pothesis 1 and $P$ under Hypothesis 2 were measured in the direction of the aftereffect 
so that they could be compared across the two directions. For $\theta$ (the estimated change in perceived position under Hypothesis 1), there was a significant main effect due to stimulus duration, $F(1,32)=4.89, p<.05$, and a significant interaction between stimulus duration and rotation rate, $F(1,32)=$ $5.52, p<.05$. For $P$, (the estimated perceived rate of rotation of the letters under Hypothesis 2), there was again a significant main effect due to stimulus duration, $F(1$, $32)=4.85, p<.05$, but no other significant effects. Similarly for $p$, the estimated probability of rotating a letter through $240^{\circ}$ instead of $120^{\circ}$, there was only a significant effect due to stimulus duration, $F(1,32)=$ $3.88, p<.05$. Table 1 shows the means.
Regardless of which hypothesis is used to describe the data, the influence of the aftereffect was greater when the stimulus duration was $2 \mathrm{sec}$ than when it was $90 \mathrm{msec}$.

In summary, the aftereffect clearly influenced the mental-rotation functions. We entertained three hypotheses as to why this might be so. Overall, the most satisfactory hypothesis was that subjects tended to rotate letters at $120^{\circ}$ from the upright through the larger $240^{\circ}$ angle when the aftereffect was in that direction. Nevertheless, there were also signs that the aftereffect caused the letters to be perceived as though displaced in the direction of the aftereffect from their true orientations. We therefore carried out a further experiment to determine whether

Table 1

Mean Percentage of Variance Accounted For and Mean Estimates of Parameters Under Each Hypothesis in Experiment 1, Shown For Each Rate of Rotation of Disk and Each Stimulus Duration

\begin{tabular}{|c|c|c|c|c|}
\hline $\begin{array}{c}\text { Rate } \\
\text { (in rpm) }\end{array}$ & $\begin{array}{l}\text { Duration } \\
\text { (in msec) }\end{array}$ & $\begin{array}{c}\% \\
\text { variance }\end{array}$ & $\begin{array}{l}\text { Mental-rotation } \\
\text { rate (in } \% / \mathrm{sec})\end{array}$ & $\begin{array}{l}\text { Parameter } \\
\text { representing } \\
\text { influence of } \\
\text { aftereffect }\end{array}$ \\
\hline
\end{tabular}

Hypothesis 1: Aftereffect influences perceived orientation

\begin{tabular}{rrrrr} 
& & & $\begin{array}{c}\theta \\
\text { (in degrees) }\end{array}$ \\
25 & 2,000 & 73.6 & 750 & 24.9 \\
25 & 90 & 78.1 & 586 & 25.8 \\
60 & 2,000 & 73.4 & 654 & 39.3 \\
60 & 90 & 72.5 & 719 & 11.9 \\
\hline
\end{tabular}

Hypothesis 2: Aftereffect summates with mental rotation

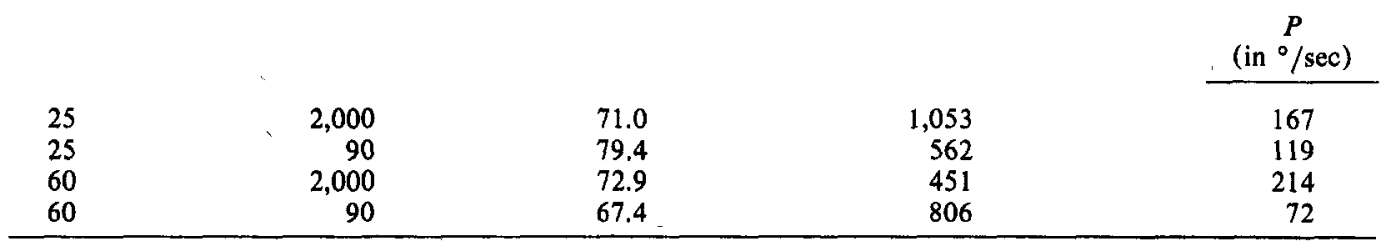

Hypothesis 3: Aftereffect induces $240^{\circ}$ rotation

\begin{tabular}{rrrrr} 
& & & $\begin{array}{r}\text { Probability of } \\
240^{\circ} \text { rotation }\end{array}$ \\
25 & & & 1,800 & 1.961 \\
25 & 2,000 & 72.2 & 699 & .634 \\
60 & 90 & 83.1 & 714 & 1.583 \\
60 & 2,000 & 80.0 & 642 & .718 \\
\hline
\end{tabular}

Note. The symbol $\theta$ refers to angular displacement; $P$ refers to the rate of aftereffect. 
subjects would in fact perceive changes in orientation of a stimulus subjected to the same motion aftereffect.

\section{Experiment 2}

It is commonly asserted that the motion aftereffect is characterized by perceived movement without perceived change in location (e.g., Kaufman, 1974, p. 403; MacKay, 1973, pp. 310-311; Rock, 1975, p. 220; Wohlgemuth, 1911, p. 88). The evidence for this seems largely introspective or anecdotal rather than objective, however. Wohlgemuth (1911, Experiment 33) did attempt a direct perceptual comparison between real movement and the aftereffect of movement, but he relied on observers' reports of what they saw rather than on a measure of discrimination; thus, in comparison with real movement, the aftereffect was said to possess a "hollow, ghost-like appearance" (p. 87). Wohlgemuth interpreted this to mean that the aftereffect was the remainder of actual movement once the component due to changing position was taken out.

MacKay (1973) has argued similarly that there are independent systems in the visual system signaling position and what he calls continuous "drift" across the retina, and that fatigue of the drift-sensitive system is responsible for the motion aftereffect (cf. Over, 1971; Sutherland, 1961): The idea that there might be a visual "channel" sensitive to movement per se is supported by evidence in mammalian visual systems for single cells responsive only to movement in particular directions (eg., Barlow \& Hill, 1963; Hubel \& Wiesel, 1965, 1968). Some illusions of movement seem to offer fairly direct evidence for a dissociation between channels signaling motion and those signaling change of location (eg., MacKay, 1980; Thorson, Lange, \& Biederman-Thorson, 1969), although these do not bear very directly on motion aftereffects per se.

Despite the evidence for a dissociation between perceived motion and perceived changes in orientation resulting from the motion aftereffect, it is conceivable that rotation might induce some change in per- ceived orientation that is independent of the motion aftereffect per se. One possible mechanism for this is optokinetically induced eye torsion. It is known that observation of a rotating stimulus can cause the eyes to rotate in the same direction (e.g., Brecher, 1934), which might cause a stationary pattern to appear tilted in the opposite direction. It is very unlikely that this would have contributed significantly to the effect obtained in Experiment 1, however. For one thing, the effect on eye torsion is small. With a large rotating disk subtending $130^{\circ}$ of visual angle, Merker and Held (1981) induced an eye torsion of about $4^{\circ}$, whereas Dichgans, Held, Young, and Brandt (1972) reported torsions of between $1^{\circ}$ and $2^{\circ}$. Howard and Templeton (1964) had subjects watch a rotating line; the maximum torsion induced was only $1.3^{\circ}$. These small effects contrast with the $25.5^{\circ}$ that would be needed to account for the effect on the mental rotation function in Experiment 1. Moreover, these eye torsions were recorded during observation of a rotating stimulus, whereas the effect in Experiment 1 was an aftereffect of rotation. There is some evidence that eye torsion induced by rotary movement is nystagmic (Brecher, 1934; Howard \& Templeton, 1964), suggesting that it would diminish fairly rapidly following cessation of the inducing stimulus.

A rotating stimulus also causes a stationary stimulus to appear tilted in the direction opposite to the rotation. This effect is larger than the effect on eye torsion and seems to depend on different processes (Merker \& Held, 1981; Wolfe \& Held, 1979). Again, however, it seems unlikely that this would have contributed appreciably to the effect observed in Experiment 1. The magnitude of the effect seems to depend on the size of the rotating stimulus; with a large disk, subtending $130^{\circ}$ of visual angle, Merker and Held (1981) induced a tilt effect of about $10^{\circ}$; but Hughes, Brecher, and Fishkin's (1972) smaller disk, subtending about $18.5^{\circ}$ of visual angle, induced a tilt effect that was less than $2^{\circ}$ at rates comparable to those of Experiment 1. Moreover, Held, Dichgans, and Bauer (1975) showed that the tilt effect declined rapidly after cessation of the rotat- 
ing stimulus, although their data do suggest some residual effect in the first seconds after exposure. In any event, according to our analysis of the results of Experiment 1, changes in perceived orientation did not constitute the most likely explanation, at least for the major part of the effect.

Even so, the data of Experiment 1 suggested that a small component of the influence on the mental-rotation function could have been due to a perceived angular displacement of the letters, especially when they were presented for the 2-sec duration. In Experiment 2, therefore, we tried to measure directly the effect of the rotating disk on subjects' judgments of orientation. The letters were replaced by an arrow; after the subjects were exposed to the disk, the arrow was presented in the six different orientations. The subjects simply made absolute judgments of the orientation of the arrow when last perceived.

\section{Method}

\section{Subjects}

The subjects were eight volunteers (four men and four women) aged 20-30 years. None had served in Experiment 1.

\section{Stimuli and Apparatus}

The stimuli consisted of an arrow, whose length matched the height of the letters used in Experiment 1 , presented at $0^{\circ}, 60^{\circ}, 120^{\circ}, 180^{\circ}, 240^{\circ}$ and $300^{\circ}$ clockwise from the vertical. The thickness of the arrow matched that of the lines from which the letters were constructed; it had a V-shaped head but no tail. It was presented within the same circular surround as were the letters in Experiment 1. The apparatus and the disk used to generate the aftereffect were also the same as in Experiment 1 .

\section{Procedure}

The subjects first judged the orientation of the arrow in each of the six orientations in the absence of any aftereffect. They were asked to make their judgments in terms of the minute hand on a clock face, so that, for instance, a true reading of the $120^{\circ}$ orientation would be " 20 minutes." They were told to estimate to the nearest minute. For these judgments, the stationary disk was presented for $5 \mathrm{sec}$, followed by a 100 -msec presentation of a dark field as a warning signal, then by a $\ 500-\mathrm{msec}$ presentation of the disk, and then the arrow for $90 \mathrm{msec}$. The field was dark for a further 2 sec; then the whole cycle was repeated. The subjects' judgments under this condition constituted a baseline.

The disk was then set in motion at $25 \mathrm{rpm}$, and after the subject had watched it for a minute or two he/she made further judgments of the orientation of the arrow when last perceived. That is, if the arrow appeared to change position, the subject was to judge its orientation in its most recently perceived position. The arrow was presented three times in each orientation at each of the two stimulus durations used in Experiment 1, namely, $2 \mathrm{sec}$ and $90 \mathrm{msec}$. The timing of disk presentation, warning signal, and stimulus presentation was the same as in Experiment 1. All subjects received 18 trialsthree presentations for each of the six orientations - at one stimulus duration and then 18 trials at the other, with the order of stimulus durations counterbalanced across subjects. The rotating disk was presented for 9 sec between trials, as in Experiment 1. The different orientations were presented in random order within each 18-trial sequence. Following this, the disk was rotated in the opposite direction, and after a period of adaptation, the sequence of 36 trials was repeated. The order of presenting the two directions of rotation was also counterbalanced across subjects.

\section{Results and Discussion}

Each subject's judgments were averaged over the three trials at each orientation for each stimulus duration and direction of the aftereffect. The baseline judgments were then subtracted from each average. For the counterclockwise aftereffect (i.e. clockwise rotation of the disk), the signs of the differences were reversed, so that all difference scores represented changes in perceived position in the direction of the aftereffect. These change scores were then subjected to ANOVA.

The overall mean was only $.38^{\prime}$, which did not differ significantly from zero, $F(1,4)=$ $2.63, n s$. There were no significant main effects or interactions. In particular there was no effect of stimulus duration $(F=.02)$. These results are therefore consistent with the widely accepted view that a pattern subjected to the motion aftereffect is seen paradoxically to move but not to change its position.

The overall mean of $.38^{\prime}$ on a clock face represents $2.28^{\circ}$, and although this was not significantly different from zero, it is reasonably consistent with what one might expect on the basis of the optokinetically induced tilt effect (e.g., Held, et al., 1975; Hughes et al., 1972). In any event, this small shift does not approach the shift of $25.33^{\circ}$ estimated according to Hypothesis 1 for the 25-rpm rotation rate in Experiment 1 . It is also well short of the shift required $\left(>30^{\circ}\right)$ 
to bring about a change in the minimum latency for $0^{\circ}$ to either $60^{\circ}$ or $300^{\circ}$ (depending on the direction of the effect), as was observed under the 2-sec stimulus duration in Experiment 1.

It might be noted that the subjects in this experiment were exposed to the rotating disk for only half the time that the subjects in Experiment 1 were exposed to it-that is, for 36 successive trials in each direction, compared with 72 in Experiment 1. This cannot explain the difference in the size of the effects in the two experiments, however. We have found that the effect of the rotating disk on the mental-rotation function does not change significantly over successive blocks of 36 trials; indeed, in unpublished research we have found this to be the case over four successive blocks of exposure to the same direction of rotation.

There remains one possibility that may save the hypothesis that the influence on the mental-rotation function was due to a perceived shift in orientation. It is conceivable that subjects do not report a change in orientation when specifically asked to focus on orientation but that they do perceive changes in orientation when the focus is on some other task, such as mental rotation. For the present, we have no data with which to test this possibility.

\section{General Discussion}

Experiment 1 showed that the aftereffect of rotary movement influences mental rotation. This influence seemed to be largely independent of the rate of rotation of the disk that induced the aftereffect. It was somewhat more pronounced when the letter to be rotated was presented for $2 \mathrm{sec}$ than when it was presented for $90 \mathrm{msec}$, but even at this short stimulus duration the effect was clearly apparent.

One explanation for the effect was that the aftereffect influenced the perceived orientation of the test letters. Overall, this hypothesis did not provide the best fit to the data, although there was some evidence that it may have contributed to the effect at the longer of the two stimulus durations. Experiment 2 showed that the aftereffect had negligible influence on subjects' reports of the perceived orientation of a stimulus. We tentatively conclude that the effect of perceived changes in orientation on the mentalrotation functions was at most slight.

If we can exclude any significant influence due to changes in perceived orientation, then our data imply an interaction between perceived and imagined movement. Thus, the equivalence between imagery and perception holds for movement as well as for position. There is still some doubt, however, as to the level of equivalence (cf. Finke, 1980). One might argue that both perceived and imagined movement converge on perceptual units dedicated to the perception of movement (MacKay's, 1973, "drift-sensitive" units, for example). This possibility, or at least a version of it, was represented by our Hypothesis 2 in Experiment 1, in which it was proposed that perceived and imagined movement might combine additively in mental rotation. This hypothesis was the least adequate in fitting the mental-rotation functions.

Alternatively, it is possible that the convergence is at a higher cognitive level. A version of this conjecture was captured by Hypothesis 3 of Experiment 1, which was that subjects were sometimes induced by the aftereffect to mentally rotate letters through the larger angle rather than the smaller one back to the upright. We supposed that this was true only when the letters were oriented at $120^{\circ}$ from the upright, and then only when the direction of the aftereffect was toward the larger $240^{\circ}$ angle. Even with this restriction, this hypothesis provided the best overall fit to the data.

Our tentative conclusion, then, is that the main influence of the aftereffect is on the subject's decision as to which way to mentally rotate the letter. We consider this conclusion preliminary, however; we plan more exacting experiments to discriminate the different hypotheses we have outlined.

\section{References}

Barlow, H. B., \& Hill, R. M. Evidence for a physiological explanation of the waterfall phenomenon and figural aftereffects. Nature, 1963, 200, 1434-1435.

Brecher, G. A.' Die optokinetische Auslösung von Augenrollung und rotatorischem Nystagmus. Pflügers Archiv für die gesamte Physiologie des Menschen und der Tiere, 1934, 234, 13-28. 
Cooper, L. A., \& Shepard, R. N. Chronometric studies of the rotation of mental images. In W. G. Chase (Ed.), Visual information processing. New York: Academic Press, 1973.

Dichgans, J., Held, R., Young, L., \& Brandt, T. Moving visual scenes influence the apparent direction of gravity. Science, 1972, 178, 1217-1219.

Finke, R. A. Levels of equivalence in imagery and perception. Psychological Review, 1980, 87, 113-132.

Held, R., Dichgans, J., \& Bauer, J. Characteristics of moving visual scenes influencing spatial orientation. Vision Research, 1975, 15; 357-165.

Howard, I. P., \& Templeton, W. B. Visually induced eye torsion and tilt adaptation. Vision Research, $1964,4,433-437$.

Hubel, D. H., \& Wiesel, T. N. Receptive fields and functional architecture in two nonstriate areas (18 and 19) of the cat. Journal of Neurophysiology, $1965,28,229-289$.

Hubel, D. H., \& Wiesel, T. N. Receptive fields and functional architecture of the monkey striate cortex. Journal of Physiology, 1968, 195, 215-243.

Hughes, P. C., Brecher, G. A., \& Físhkin, S. M. Effects of rotating backgrounds upon the perception of verticality. Perception \& Psychophysics, 1972, 11, 135138.

Kaufman, L. Sight and mind: An introduction to visual perception. London: Oxford University Press, 1974.

MacKay, D. M. Visual stability and voluntary eye movements. In R. Jung (Ed.), Handbook of sensory physiology (Vol. III/3A). Heidelberg, West Germany: Springer-Verlag, 1973.

MacKay, D. M. Illusory reversal of extrafoveally perceived displacement. Nature, 1980, 284, 257.

Merker, B. H., \& Held, R. Eye torsion and the apparent horizon under head tilt and visual field rotation. $\mathrm{Vi}$ sion Research, 1981, 21, 543-547.

Over, R. Comparison of normalization theory and neural enhancement explanation of negative aftereffects. Psychological Bulletin, 1971, 75, 225-243.

Rock, I. An introduction to perception. New York: Macmillan, 1975.

Shepard, R. N., \& Metzler, J. Mental rotation of threedimensional objects. Science, 1971, 171, 701-703.

Sutherland, N. S. Figural aftereffects and apparent size. Quarterly Journal of Experimental Psychology, 1961, 13, 222-228.

Thorson, J., Lange, G. D., \& Biederman-Thorson, M. Objective measure of the dynamics of a visual movement illusion. Science, 1969, 164, 1087-1088.

Winer, B. J. Statistical principles in experimental design. New York: McGraw-Hill, 1971.

Wohlgemuth, A. On the aftereffect of seen movement. British Journal of Psychology Monograph Supplement, $1911,1,1-117$.

Wolfe, J. M. \& Held, R. Eye torsion and visual tilt are mediated by different binocular processes. Vision Research, 1979, 19, 917-920.

Received March 30, 1981 